\title{
The Kac limit for finite-range spin glasses
}

\author{
Silvio Franz $z^{\ddagger}$, Fabio Lucio Toninelli ${ }^{\dagger}$ \\ ¥ The Abdus Salam International Center for Theoretical Physics, Condensed Matter Group \\ Strada Costiera 11, P.O. Box 586, I-34100 Trieste, Italy \\ † EURANDOM, Technische Universiteit Eindhoven, P.O. Box 513, 5600 MB Eindhoven, The Netherlands
}

(November 3, 2018)

\begin{abstract}
We consider a finite range spin glass model in arbitrary dimension, where the strength of the two-body coupling decays to zero over some distance $\gamma^{-1}$. We show that, under mild assumptions on the interaction potential, the infinite-volume free energy of the system converges to that of the Sherrington-Kirkpatrick one, in the Kac limit $\gamma \rightarrow 0$. This could be a first step toward an expansion around mean field theory, for spin glass systems.
\end{abstract}

05.20.-y, $75.10 \mathrm{Nr}$

Despite years of debate, the nature of the spin glass phase of the finite dimensional systems remains a major open problem in statistical physics. Two competing theories have been proposed as candidate to explain spin glass physics at low temperature: the theory of replica symmetry breaking [1] [2] and the droplet theory [3] [4]. The former, based on the analysis of the long range SherringtonKirkpatrick (SK) spin glass, predicts a rich phenomenology with ergodicity breaking not related to any physical symmetry breaking and susceptibility anomalies related to the presence on many pure states. The latter assimilates spin glasses to some kind of "disguised ferromagnet" -albeit with complex phenomenology- where the transition appears as a conventional symmetry breaking phenomenon. Both theories being non-rigorous in the applications to finite dimensional systems, it appears very difficult to solve the question on a purely theoretical ground. On the other hand, experiments in 3D and numerical simulations in 3 and $4 \mathrm{D}$ fail to give compelling evidence in favour of one or the other of the two theories: the times probed in the experiments are too short to settle the question of the presence or absence of replica symmetry breaking and the related issue of asymptotic existence of response anomalies during aging dynamics, and the length scales probed in the simulations are too small to infer the behaviour of the thermodynamic limit. Rigorous analysis of finite dimensional systems turns out to be very hard, and so far has not been able to exclude either scenario, although it has produced [5] considerable conceptual clarification, and shown some of the subtleties hidden even in the definition of the infinite volume limit of these models. Even at the mean field level, only very recently, simple interpolation methods have been introduced [6] [7] [8] which have allowed to prove [9] the Parisi solution for the SK model. Interpolation methods have subsequently been applied also in the context of finite range spin glasses, e.g. in [10].

In this Letter we focus our attention on the Kac limit of finite range spin glasses as first considered in [11], and later studied in [12] and [13]. Kac models are a classical tool of mathematical physics, where one considers variables interacting via a potential with finite range $\xi=\gamma^{-1}$, which tends to infinity after the thermodynamic limit is taken. In a classical paper [14] Penrose and Lebowitz proved that for conventional non-disordered systems, the free-energy tends (modulo the Maxwell construction) to the one of the corresponding mean-field system where the interactions do not decay with distance and scale with the size of the system. We combine here the idea of the interpolating model with the idea [14] of dividing the system into boxes of suitable size to prove the same property in spin glasses.

Other disordered models with Kac-type interactions have been studied in previous literature. For instance, see [15] and references therein for the case of the Hopfield model.

The model we consider is defined on the $d$-dimensional lattice $Z^{d}$, with Ising spin degrees of freedom $\sigma_{i}= \pm 1, i \in$ $Z^{d}$. Given a finite hypercube $\Lambda$ of side $L$ one defines the finite volume Hamiltonian as

$$
H_{\Lambda}^{(\gamma)}(\sigma, h ; J)=-\sum_{i, j \in \Lambda} \sqrt{\frac{w(i-j ; \gamma)}{2 W(\gamma)}} J_{i j} \sigma_{i} \sigma_{j}-h \sum_{i \in \Lambda} \sigma_{i},
$$

where $W(\gamma)=\sum_{i \in Z^{d}} w(i ; \gamma)$ and $w(r ; \gamma)=\gamma^{d} \phi(\gamma r)$ for some smooth, nonnegative function $\phi(r)$, decaying sufficiently fast for $|r| \rightarrow \infty$ to have $W(\gamma)<\infty$. The parameter $\gamma=\xi^{-1}$ is the inverse range of the interaction. The quenched couplings $J_{i j}$ are i.i.d. Gaussian $N(0,1)$ variables, and we denote by $E$ the corresponding averages. As is well known [16] [17], the infinite-volume limit of the quenched free energy

$$
f^{(\gamma)}(\beta, h)=-\lim _{L \rightarrow \infty} \frac{1}{\beta|\Lambda|} E \ln Z_{\Lambda}^{(\gamma)}(\beta, h ; J)
$$

exists.

On the other hand, the Hamiltonian of the SK spin glass mean field model is defined as [18]

$$
H_{|\Lambda|}^{S . K .}(\sigma, h ; J)=-\frac{1}{\sqrt{2|\Lambda|}} \sum_{i, j \in \Lambda} J_{i j} \sigma_{i} \sigma_{j}-h \sum_{i \in \Lambda} \sigma_{i},
$$


where $|\Lambda|=L^{d}$ is the number of lattice sites in $\Lambda$. Subadditivity of the corresponding free energy and existence of its infinite volume limit

$$
f^{S . K .}(\beta, h)=-\lim _{L \rightarrow \infty} \frac{1}{\beta|\Lambda|} E \ln Z_{|\Lambda|}^{S . K .}(\beta, h ; J)
$$

has been proven in [6].

It was recently shown in [13] that the free energy of model (1) is bounded below by that of SK:

$$
f^{(\gamma)}(\beta, h) \geq f^{S \cdot K \cdot}(\beta, h)
$$

for any value of $d, \beta, h$ and $\gamma$, provided that the potential $\phi(i-j)$ is nonnegative definite, i.e., its Fourier transform is nonnegative. For instance, it is immediate to check this condition for $\phi(i-j)=e^{-\sum_{\alpha=1}^{d}\left|i_{\alpha}-j_{\alpha}\right|}$, which for $d=1$ is just the potential considered originally by Kac in [19]. In the present paper, we provide the complementary bound, which allows to fully characterize the quenched free energy in the Kac limit $\gamma \rightarrow 0$ :

Theorem 1 Assume that $\sum_{i \in Z^{d}} \phi(i)<\infty$. Then, for any $\beta$ and $h$ one has

$$
\lim _{\gamma \rightarrow 0} f^{(\gamma)}(\beta, h) \leq f^{S . K \cdot}(\beta, h) .
$$

If in addition all the Fourier components of $\phi$ are nonnegative, then

$$
\lim _{\gamma \rightarrow 0} f^{(\gamma)}(\beta, h)=f^{S . K \cdot}(\beta, h) .
$$

Together with Talagrand's recently established proof [9] of the Parisi ansatz for the SK model, this shows that the Parisi theory [1] gives the correct free energy for finite dimensional spin glasses in the Kac limit.

The idea of the proof is to interpolate between the Kac model in a volume $|\Lambda|$ and a system made of a collection of many independent SK subsystems of volume $M=\ell^{d}$. The crucial point, as in [14], is to choose

$$
\ell \ll \xi \ll L,
$$

and to let the three lengths diverge in this order. Let us divide the box $\Lambda$ into sub-cubes $\Omega_{n}$ of volume $M, n=$ $1, \cdots,|\Lambda| / M$, and introduce the interpolating partition function

$$
\begin{aligned}
Z_{\Lambda}(t)= & \sum_{\sigma} \exp \left(\beta \sqrt{1-t} \sum_{n} \sum_{i, j \in \Omega_{n}} \frac{J_{i j}}{\sqrt{2 M}} \sigma_{i} \sigma_{j}\right) \\
& \times \exp \left(\beta \sqrt{t} \sum_{i, j \in \Lambda} \sqrt{\frac{w(i-j ; \gamma)}{2 W(\gamma)}} J_{i j}^{\prime} \sigma_{i} \sigma_{j}+\beta h \sum_{i \in \Lambda} \sigma_{i}\right),
\end{aligned}
$$

$$
\begin{aligned}
& \frac{1}{|\Lambda|} E \ln Z_{\Lambda}(0)=\frac{1}{M} E \ln Z_{M}^{S . K .} \cdot(\beta, h ; J) \\
& \frac{1}{|\Lambda|} E \ln Z_{\Lambda}(1)=\frac{1}{|\Lambda|} E \ln Z_{\Lambda}^{(\gamma)}(\beta, h ; J) .
\end{aligned}
$$

As we show below, one has

$$
\lim _{\gamma \rightarrow 0} \lim _{L \rightarrow \infty} \frac{d}{d t} \frac{1}{|\Lambda|} E \ln Z_{\Lambda}(t) \geq 0
$$

uniformly for $0 \leq t \leq 1$. After integration on $t$ between 0 and 1 and taking the large $M$ limit, one finds therefore the desired result

$$
\begin{aligned}
-\beta \lim _{\gamma \rightarrow 0} f^{(\gamma)}(\beta, h) & \geq \lim _{M \rightarrow \infty} \frac{1}{M} E \ln Z_{M}^{S . K} \cdot(\beta, h ; J) \\
& =-\beta f^{S . K .}(\beta, h) .
\end{aligned}
$$

Denoting as $\langle$.$\rangle the Gibbs average, the computation of$ the $t$ derivative gives, up to terms negligible for large $L$,

$$
\begin{aligned}
\frac{d}{d t} \frac{1}{|\Lambda|} E \ln Z_{\Lambda}(t)= & \frac{\beta^{2}}{4|\Lambda|} E\left[\sum_{n} \sum_{i, j \in \Omega_{n}} \frac{1}{M}\left\langle\sigma_{i} \sigma_{j}\right\rangle^{2}\right. \\
& \left.-\sum_{i, j \in \Lambda} \frac{w(i-j ; \gamma)}{W(\gamma)}\left\langle\sigma_{i} \sigma_{j}\right\rangle^{2}\right],
\end{aligned}
$$

where we have used integration by parts on the Gaussian disorder and the property

$$
\lim _{L \rightarrow \infty} \frac{1}{|\Lambda|} \sum_{i, j \in \Lambda} \frac{w(i-j ; \gamma)}{W(\gamma)}=1 .
$$

Introducing two replicas with identical quenched couplings and spin configurations $\sigma^{1}, \sigma^{2}$, we can write (13) as:

$$
\begin{aligned}
\frac{d}{d t} \frac{1}{|\Lambda|} E \ln Z_{\Lambda}(t)= & \frac{\beta^{2}}{4|\Lambda|} E\left[\sum_{n} \frac{1}{M} \sum_{i, j \in \Omega_{n}}\left\langle\sigma_{i}^{1} \sigma_{i}^{2} \sigma_{j}^{1} \sigma_{j}^{2}\right\rangle\right. \\
& \left.-\sum_{i, j \in \Lambda} \frac{w(i-j ; \gamma)}{W(\gamma)}\left\langle\sigma_{i}^{1} \sigma_{i}^{2} \sigma_{j}^{1} \sigma_{j}^{2}\right\rangle\right] .
\end{aligned}
$$

Denoting the partial overlap in the $n$-th sub-cube as $q_{12}^{(n)}=1 / M \sum_{i \in \Omega_{n}} \sigma_{i}^{1} \sigma_{i}^{2}$, the first term of the r.h.s. can be rewritten as

$$
\frac{\beta^{2} M}{4|\Lambda|} \sum_{n} E\left\langle\left(q_{12}^{(n)}\right)^{2}\right\rangle .
$$

As for the second term, defining

$$
w_{m n}^{+}=\sup _{i \in \Omega_{m}, j \in \Omega_{n}} \frac{w(i-j ; \gamma)}{W(\gamma)}
$$

where the Gaussian variables $J^{\prime}$ are independent of the $J$. Note that and using the straightforward inequality $2 x y \leq x^{2}+y^{2}$, one has 


$$
\begin{aligned}
& \frac{1}{|\Lambda|} \sum_{i, j \in \Lambda} \frac{w(i-j ; \gamma)}{W(\gamma)} E\left\langle\sigma_{i}^{1} \sigma_{i}^{2} \sigma_{j}^{1} \sigma_{j}^{2}\right\rangle \\
& \leq \frac{M^{2}}{2|\Lambda|} \sum_{n, m} w_{m n}^{+} E\left\langle\left(q_{12}^{(n)}\right)^{2}+\left(q_{12}^{(m)}\right)^{2}\right\rangle .
\end{aligned}
$$

In the Kac limit $\gamma \rightarrow 0$, the diagonal terms $n=m$ give a vanishing contribution. As for the nondiagonal ones, one observes that

$$
\lim _{\gamma \rightarrow 0} \sum_{m(\neq n)} w_{m n}^{+}=\frac{1}{M}
$$

where the summation runs only on one of the two indices, so that finally the r.h.s. of (18) is bounded above by

$$
\frac{M}{|\Lambda|} \sum_{n} E\left\langle\left(q_{12}^{(n)}\right)^{2}\right\rangle
$$

apart from a negligible error term. Together with Eqs. (15) and (16), this proves (11) and therefore the Theorem.

As a side remark, it is easy to employ this method, together with that of [13], to obtain a new proof of the existence of the thermodynamic limit for the SK model, independent of the convexity argument developed in [6].

It is possible to generalize this theorem to the "diluted Kac spin glass" case [13] where each given spin $\sigma_{i}$ interacts with a finite random number of other spins $\sigma_{j}$, which are chosen randomly according to a probability distribution that decays to zero on the scale $\xi$, as $|i-j|$ diverges. In the Kac limit $\xi \rightarrow \infty$, one can prove that the free energy of the model converges to that of its mean field counterpart, which in that case is the Viana-Bray model [20]. Full details of the proof are given in [21].

A second generalization of our result is to consider two replicas of the system, coupled via a term depending on their mutual overlap. This problem has been considered for instance in [22] and is relevant for the study of glassy dynamics, especially if applied to models which exhibit "one-step replica symmetry breaking" [1]. The new feature here is that, at the mean field level, the free energy of the coupled system can be expressed [22] in terms of an effective potential depending on the overlap, which turns out to be nonconvex. It was argued in [23] that a minimal modification of the theory in finite dimension requires restoration of the convexity through the Maxwell construction. This, analogously to the ordered case [14], emerges naturally in the Kac limit of finite range models. We plan to report on this soon [24].

The main interest of the result presented in this Letter is that it could represent for spin glasses, a first step toward an expansion around the mean field case, which would hopefully shed some light on the nature of the spin glass phase for models with finite -albeit large- interaction range. This hope is supported by the fact that a similar program has been successfully carried on recently for non-random ferromagnetic spin systems [25] [26] [27] and continuous particle systems [28], showing that in dimension $d \geq 2$ it is possible to write a controlled expansion around the $\gamma=0$ point, and to prove rigorously that for large but finite $\xi$ the system has a phase transition (broken spin flip or liquid-vapor, respectively) with coexisting phases.

\section{Acknowledgments}

We would like to thank Francesco Guerra for many enlightening conversations. F.L.T. is grateful to the Condensed Matter Group of the ICTP for kind hospitality during the preparation of this work. This work was supported in part by the European Community's Human Potential programme under contract "HPRN-CT-200200319 STIPCO".

$\ddagger$ e-mail: franz@ictp.trieste.it

$\dagger$ e-mail: ftoninel@euridice.tue.nl

[1] M. Mézard, G. Parisi and M.A. Virasoro, Spin glass theory and beyond, World Scientific, Singapore (1987).

[2] E. Marinari, G. Parisi, F. Ricci-Tersenghi, J. RuizLorenzo, F. Zuliani, J. Stat. Phys. 98, 973 (2000).

[3] D.S. Fisher, D.A. Huse, Phys. Rev. Lett. 56, 1601-1604 (1986).

[4] A.J. Bray and M.A. Moore, in Heidelberg Colloquium on Glassy Dynamics, eds. J.L. Van Hemmen and I. Morgenstern, Springer-Verlag (1986) p. 121.

[5] C.M. Newman, D.L. Stein, J. Phys.: Condens. Matter 15, R1319-R1364 (2003).

[6] F. Guerra, F.L. Toninelli, Commun. Math. Phys. 230 (1), 71-79 (2002).

[7] F. Guerra, Commun. Math. Phys. 233 (1), 1-12 (2003).

[8] M. Aizenman, R. Sims, S.L. Starr, to appear. Preprint cond-mat/0306386.

[9] M. Talagrand, C. R. Acad. Sci. Paris, Ser. I 337, 111-114 (2003).

[10] P. Contucci, S. Graffi, J. Stat. Phys, to appear. Preprint math-ph/0302013.

[11] J. Fröhlich, B. Zegarlinski, Commun. Math. Phys. 112, 553-566 (1987).

[12] A. Bovier, J. Stat. Phys. 91, 459-474 (1998).

[13] F. Guerra, F.L. Toninelli, J. Phys. A 36 (43), 1098710995 (2003).

[14] J.L. Lebowitz, O. Penrose, J. Math. Phys. 7 (1), 98-113 (1966).

[15] A. Bovier, V. Gayrard, P. Picco, in Mathematical Aspects of Spin glasses and Neuronal Networks, eds. A. Bovier and P. Picco, Progress in Probability 41, Birkhäuser (1998) pp. 3-89.

[16] P.A. Vuillermot, J. Phys. A 10, 1319-1333 (1977).

[17] A.C.D. van Enter, J.L. van Hemmen, J. Stat. Phys. 32, 141 (1983).

[18] D. Sherrington, S. Kirkpatrick, Phys. Rev. Lett. 35, 1792-1796 (1975). 
[19] M. Kac, Phys. Fluids 2, 8 (1959).

[20] L. Viana, A. J. Bray, J. Phys. C 18, 3037 (1985).

[21] S. Franz, F.L. Toninelli, The Kac limit for diluted spin glasses, to appear.

[22] S. Franz, G. Parisi, J. Phys. I (France) 5, 1401 (1995).

[23] S. Franz, G. Parisi, Phys. Rev. Lett. 79, 2486 (1997); Physica A 261, 317 (1998).

[24] S. Franz, F. L. Toninelli, in preparation

[25] M. Cassandro, E. Presutti, Markov Proc. Rel. Fields 2, 241 (1996).

[26] A. Bovier, M. Zahradník, J. Stat. Phys. 87, 311-333 (1997).

[27] T. Bodineau, E. Presutti, Commun. Math. Phys. 189, 287-298 (1997).

[28] J.L. Lebowitz, A. E. Mazel, E. Presutti, Phys. Rev. Lett. 80, 4701-4704 (1998); J. Stat. Phys. 94, 955-1025 (1999). 